\title{
Dynamical changes of the polar cap potential structure: an information theory approach
}

\author{
I. Coco, G. Consolini, E. Amata, M. F. Marcucci, and D. Ambrosino \\ INAF-Istituto di Fisica dello Spazio Interplanetario, Via Fosso del Cavaliere, 100, 00133 Roma, Italy \\ Received: 28 February 2011 - Revised: 3 October 2011 - Accepted: 5 October 2011 - Published: 17 October 2011
}

\begin{abstract}
Some features, such as vortex structures often observed through a wide spread of spatial scales, suggest that ionospheric convection is turbulent and complex in nature. Here, applying concepts from information theory and complex system physics, we firstly evaluate a pseudo Shannon entropy, $H$, associated with the polar cap potential obtained from the Super Dual Auroral Radar Network (SuperDARN) and, then, estimate the degree of disorder and the degree of complexity of ionospheric convection under different Interplanetary Magnetic Field (IMF) conditions. The aforementioned quantities are computed starting from time series of the coefficients of the 4th order spherical harmonics expansion of the polar cap potential for three periods, characterised by: (i) steady IMF $B_{z}>0$, (ii) steady IMF $B_{z}<0$ and (iii) a double rotation from negative to positive and then positive to negative $B_{z}$. A neat dynamical topological transition is observed when the IMF $B_{z}$ turns from negative to positive and vice versa, pointing toward the possible occurrence of an order/disorder phase transition, which is the counterpart of the large scale convection rearrangement and of the increase of the global coherence. This result has been confirmed by applying the same analysis to a larger data base of about twenty days of SuperDARN data, allowing to investigate the role of IMF $B_{y}$ too.
\end{abstract}

\section{Introduction}

High-latitude ionospheric convection is the result of direct coupling between the solar wind and the Earth's magnetosphere. The magnetospheric electric fields map down in the ionosphere giving rise to a motion of ions and electrons in the $\boldsymbol{E} \times \boldsymbol{B}$ direction: this results in a multi-cell pattern in

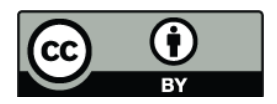

Correspondence to: I. Coco

(igino.coco@ifsi-roma.inaf.it) the polar cap that is a "mirror" on a reduced space scale of the magnetospheric plasma motion. It is now well accepted that the most important parameter, which drives the dynamical processes in the magnetosphere-ionosphere system and the ionospheric convection pattern configuration, is the Interplanetary Magnetic Field (IMF), whose orientation with respect to the Earth's magnetic field is crucial for the amount of energy, momentum and plasma particles that can penetrate the magnetospheric cavity. When a quasi steady IMF $B_{z}<0$ component is present (southward IMF $B_{z}$ ), reconnection is thought to take place at the subsolar magnetopause, creating open field lines which are dragged towards the tail, where they reconnect again, pushing plasma back to the dayside magnetosphere along the flanks: the ionospheric convection is organised in a two-cell pattern, where plasma is flowing antisunward at high latitudes in the polar cap, and sunward at lower latitudes (Dungey convection cycle: e.g. Dungey, 1961). When IMF is dominated by a $B_{z}>0$ component (northward IMF $B_{z}$ ), reconnection is favoured tailward of the polar cusps: now the open field lines are pushed sunward by the magnetic tension, and again a double cell pattern should appear in the polar caps, but in this case plasma flows sunward at very high latitudes and antisunward at lower latitudes (e.g. Burke et al., 1979; Huang et al., 2000). Reconnection in the tail can always occur due to the substorm cycle or unbalanced reconnection processes in the far tail, giving rise to one or more convection cells in the nightside even for northward IMF $B_{z}$; moreover, viscous processes at the magnetopause can produce elongated convection cells in the dusk and dawn ionosphere, that can be more evident when the Dungey convection cycle for southward IMF $B_{z}$ is not established (e.g. Axford and Hines, 1961; Cowley and Lockwood, 1992). In summary, the ionospheric convection for northward IMF $B_{z}$ is far less homogeneous than in the opposite case, showing the emergence of more than two convection cells due to several different competing mechanisms. The scheme is further complicated by the IMF $B_{y}$ component, which acts on the

Published by Copernicus Publications on behalf of the European Geosciences Union and the American Geophysical Union. 
symmetry of the convection patterns, rotating the cell system towards dawn or dusk according to the sign of $B_{y}$; in the limit of $\left|B_{y}\right| \gg\left|B_{z}\right|$ usually only one big convection cell is observed (e.g. Reiff and Burch, 1985; Gosling et al., 1990; Ruohoniemi and Greenwald, 1996, 2005).

On the other hand, evidences exist that the Earth's magnetosphere/ionosphere can be viewed as a physical system operating in a non-equilibrium dynamical state and displaying dynamical complexity (e.g. Sharma and Kaw, 2005; Consolini et al., 2008). Following Chang et al. (2006), dynamical complexity can be defined as "a phenomenon exhibited by a nonlinear interacting dynamical system within which multitudes of different sizes of large scale coherent structures are formed, resulting in a global nonlinear stochastic behavior for the dynamical systems, which is vastly different from what could be surmised from the original dynamical equations". In other words, complexity often shows up as the tendency of a non equilibrium system to display a certain degree of spatio-temporally coherent features resulting from the competition of different basic spatial patterns playing the role of interacting subunits. It is important to remark that complexity requires the occurrence of nonlinearities and the intertwining of order and disorder (Nicolis and Nicolis, 2007) and that it is generally related to the emergence of selforganisation in open systems (Klimontovich, 1991, 1996).

In the last two decades, the evidence of large scale coherence and multiscale nature of magnetospheric dynamics was clearly recognised in different ways, analysing both the low-dymensional behaviour of the large scale dynamics and the turbulent and critical nature of the small scale processes (e.g. Chang, 1992; Consolini et al., 1996; Klimas et al., 1996; Sharma and Kaw, 2005). In particular, the emergence of large scale coherence during magnetic substorms was interpreted and modelled in terms of first-order phase transitions in out-of-equilibrium dynamical systems (Sitnov et al., 2000, 2001). The increase of coherence during magnetospheric disturbed periods is the signature of the emergence of longrange space-time correlation, and, to some extent, of large scale self-organisation.

The Super Dual Auroral Radar Network (SuperDARN, Greenwald et al., 1995; Chisham et al., 2007) is nowadays one of the most important instruments to reconstruct and monitor the high-latitude ionospheric convection. The SuperDARN radars measure the Doppler shift of field-aligned density irregularities in the ionosphere which drift following the motion of the ambient plasma. The line of sight velocities of each radar are combined together using a technique described in Ruohoniemi and Baker (1998), allowing to reconstruct the isocontours of the Polar Cap Potential (PCP), which is closely related to the energy transfer from the solar wind to the magnetosphere and the ionosphere systems. A number of studies have now demonstrated that the maximum variations of the PCP on the dayside and on the nightside are equivalent to the reconnection rates, i.e. the rate of transfer of magnetic flux across unit length of the separatrix between unreconnected and reconnected field lines (e.g. Chisham et al., 2008, and references therein).

Recently, several authors studied ionospheric convection in the framework of complex systems, using SuperDARN data. Among others, Abel et al. (2009) found that turbulent features typical of the solar wind are present in the highlatitude ionosphere in regions where open field lines map, and that the degree of intermittency is controlled by the IMF clock angle, while Parkinson $(2006,2008)$ found a complex scaling of the convection velocity fluctuations in the F-region ionosphere.

In this paper we aim at extending the idea of global first order phase transition to the magnetosphere-ionosphere coupled system, by evidencing the emergence of coherence and self-organisation in the high-latitude ionosphere during the increase of magnetospheric convection. For that purpose, we will concentrate on large scale features of ionospheric convection, such as vortices and cell-like convection patterns, whose configuration is modulated by the IMF, and will use concepts taken from information theory applied to the polar cap potential, in order to build up a measure of complexity in the way already followed by Shiner et al. (1999) and Consolini et al. (2009). In particular, we aim at quantifying the spatial complexity of the polar cap potential and its dependence on the orientation of the IMF.

The paper is structured as follows: in Sect. 2, in the framework of information theory and complexity, we will define a pseudo Shannon entropy, $H$, the time dependent disorder degree, $\Delta(t)$, and the Second Order Complexity Measure, $\Gamma_{11}$; in Sect. 3 the Ruohoniemi and Baker (1998) technique for obtaining the polar cap potential will be described in some more detail, and the application of the information theory concepts to the SuperDARN data will be introduced; in Sect. 4 we will first show the results for a couple of case studies where the IMF is steadily southward/northward directed, and a case study where IMF $B_{z}$ is varying throughout the event (Sect. 4.1), evidencing how ordered/disordered configurations are concentrated in periods of southward/northward IMF and how complexity shows up in "intermediate" states; then, in Sect. 4.2, we will analyze a larger data base of about twenty days of SuperDARN data during February 2002, encompassing all possible IMF configurations and investigating the effect of IMF $B_{y}$ on the emergence of complexity. Conclusions are drawn in Sect. 5.

\section{Shannon entropy and complexity: a brief introduction}

As shown in several contexts, the investigation of complex dynamics may benefit from the application of concepts developed in the framework of information theory (e.g. Haken, 2004; Nicolis and Nicolis, 2007). Indeed, the information theory methods, based on probability theory and statistics concepts, allow a different approach to the dynamics of 
complex systems, characterised by a multitude of interacting scales, approach which is able to extract and characterise some common and universal features of complex systems. For instance, Shannon entropy, mutual information and transfer entropy have been successfully applied to investigate the occurrence of phase transitions in several dynamical complex systems, ranging from the flocking model (Wicks et al., 2007), to physiology (Quian Quiroga et al., 2000), to the solar cycle (Sello, 2000, 2003; Consolini et al., 2009), to the evolution of the geomagnetic field (De Santis et al., 2004), to magnetospheric dynamics (Chen et al., 2008; De Michelis et al., 2011).

One of the major results of information theory surely stands on the concept of information entropy, originally introduced by Claude Shannon (Shannon, 1948) and for this reason also named Shannon entropy. This quantity is defined as follows:

$S_{I}=-\sum_{i=1}^{N} p_{i} \log _{2}\left(p_{i}\right)$

where $p_{i}$ is the probability of observing a certain state (configuration or value) over a set of $N$ possible states (configurations or values). In the framework of signal analysis the Shannon entropy, $S_{I}$, represents the average amount of code length needed to encode a randomly chosen value. However, it can be considered as a measure of the information/uncertainty content in a certain distribution of states (configurations or values). Furthermore, on the basis of the definition given in Eq. (1), the Shannon entropy, $S_{I}$, takes its largest value $S_{I}^{\max }=\log _{2} N$ in the case of equiprobable distributions $\left(p_{i}=1 / N\right)$ and its smallest value $S_{I}^{\min }=0$ for $\delta$-like distributions. The Shannon entropy, $S_{I}$, also satisfies all the properties of additivity and convexity, required by a properly defined entropy.

Although the original concept of Shannon entropy was introduced to characterise the information content of a sequence of discrete and independent random variables having $N$ possible outcomes $x_{i}(i=1, \ldots, N)$ with associated probabilities $p_{i}$, in the course of the years the definition of Shannon entropy $S_{I}$ has been also extended to those situations in which the quantities $p_{i}$ are not probabilities of outcomes but statistical and/or relative weights of an observable (e.g. Quian Quiroga et al., 2000; Sello, 2000; De Santis et al., 2004). For instance, Quian Quiroga et al. (2000) defined $p_{i}$ as the normalised spectral density at the frequency $\omega_{i}$, i.e. $p_{i}=S\left(\omega_{i}\right) / \sum_{i} S\left(\omega_{i}\right)$, which is analogous to a relative weight.

The application of Shannon entropy to the case of statistical weights relies on the mathematical definition of probability in terms of a positive defined Lebesgue measure $\mu(A)$ normalised to 1. For instance, in the Feller's book (Feller, 1970), page 22, we read: "Given a discrete sample space $\mathcal{E}$ with sample points $E_{1}, E_{2}$, . . ., we shall assume that with each point $E_{j}$ there is associated a number, called probability of $E_{j}$ and denoted by $P\left\{E_{j}\right\}$. It is to be nonnegative and such that $P\left\{E_{1}\right\}+P\left\{E_{2}\right\}+\ldots=1$." Furthermore, the assumption of $p_{i}$ 's as relative statistical weights of spectral densities in different frequency domains is quite common also in the investigation of self-organisation in farfrom-equilibrium open systems (e.g. Klimontovich, 1995).

In the following of this paper, we will replace the $p_{i}$ probabilities in Eq. (1) by the relative weights of given eigenfunctions of an observable, $w_{i}$, so as to build an analogue of the Shannon entropy, that we call pseudo Shannon entropy, $H$. Because $H$ attains its maximum value, $H^{\max }=\log _{2}(N)$, for a uniform distribution (i.e. the case of $w_{i}=1 / N$, where $N$ is the number of eigenfunctions considered), the definition of the following normalised quantity turns to be helpful in comparing different configurations of a dynamical system:

$\Delta=\frac{H}{H^{\max }}=-\frac{1}{\log _{2}(N)} \sum_{i=1}^{N} w_{i} \log _{2}\left(w_{i}\right)$,

which can attain values in the interval $[0,1]$.

Because the minimum and maximum values of $H$ correspond to the two extreme situations of monochromatic (single mode) spectra ( $\delta$-like distribution of $w_{i}$ ) and flat spectra (uniform distribution with equiprobable $w_{i}$ ), the values $\Delta=0$ and $\Delta=1$ can be associated with maximum order and maximum disorder of the system described by the given eigenfunctions. The quantity $\Delta$ was originally introduced by Landsberg (1978) (see also Landsberg, 1984) and provides a measure of the degree of disorder. From definition of Eq. (2) it follows that a measure of order (degree of order) is $\Omega=1-\Delta$. Because in the case of a nonequilibrium system a measure of the distance from equilibrium is provided by the quantity $S-S_{\text {eq }}$ (Ebeling and Klimontovich, 1984; Klimontovich, 1995, 1996), where $S$ and $S_{\text {eq }}$ are the entropies of the nonequilibrium and of the equilibrium states respectively, and because in statistical thermodynamics the state of maximum entropy is the equilibrium one, it follows that $\Omega=\left(S_{\text {eq }}-S\right) / S_{\text {eq }}$ really represents a measure of order in terms of normalised distance from equilibrium.

Several attempts have been made in the past in order to characterise the degree of complexity and/or selforganisation in non-equilibrium systems. Among the wide variety of definitions, one particularly simple and easy to compute was introduced by Landsberg and Shiner (1998) and Shiner et al. (1999). This complexity measure, $\Gamma_{11}$, is defined as

$\Gamma_{11}=\Delta \cdot \Omega=\Delta(1-\Delta)=\Omega(1-\Omega)$,

which is a special case of more general complexity measures $\Gamma_{\alpha \beta}=\Delta^{\alpha} \Omega^{\beta}$. According to the above definition, the complexity measure $\Gamma_{11}$ implies that a non-vanishing level of complexity is possible only in nonequilibrium systems with some less than maximal order (Shiner et al., 1999). Although to a first reading one could conclude that all the required information on the complex nature of the observed 
phenomenon is contained in the degree of disorder $\Delta$ (or equivalently in the degree of order $\Omega$ ) this is not correct. Indeed, one way to define complexity is that it mainly arises in those situations where there is a certain competition between order and disorder. This is the reason for defining as a measure of complexity the product of order and disorder, i.e. a quantity which is not a linear function of order or disorder. In this framework, the measure of complexity $\Gamma_{11}$ and the disorder measure $\Delta$ provide complementary information. We remark that according to the definition of $\Gamma_{11}$, the same degree of complexity can be found for situations in which the degree of disorder or that of order dominates. However, the emerging of complexity achieves a different meaning in the two situations. Thus, a better characterization of the complex nature of the system arises from the specification of its state in the $\left\{\Delta, \Gamma_{11}\right\}$-space.

\section{Data analysis methodology}

SuperDARN radars work in "common" operating mode for most of the time, which means that they are synchronized over the whole polar cap, giving one- and two-minutes time series of the line-of-sight (l.o.s.) ionospheric convection velocity as a function of magnetic latitude and longitude. The l.o.s. velocity is just a component of the actual velocity vector in the direction along which the radar points during a given time interval. To reconstruct the whole velocity field over the polar cap, so that it matches as closely as possible the "true" ionospheric convection, all measurements in the radar reference frames are first averaged and median filtered over a common spatial grid of approximately $1^{\circ} \times 1^{\circ}$ Altitude Adjusted Corrected Geomagnetic coordinates (AACGM, Baker and Wing, 1989). Successively, to fill the coverage gaps of the radar fields of view, a certain amount of model data is uniformely added up: these data come from empirical models built over years of SuperDARN measurements and are chosen to represent the average convection patterns expected for the IMF configuration at the given ttime (Ruohoniemi and Greenwald, 1996, 2005). The technique by Ruohoniemi and Baker (1998) aims to reconstruct the isocontours of the PCP, $\Phi$, at time $t$, through a spherical harmonics expansion as follows:

$\Phi(\theta, \varphi ; t)=\Re e\left\{\sum_{l=0}^{\infty} \sum_{m=0}^{l} c_{l m}(t) \exp (\operatorname{im} \varphi) P_{l}^{m}(\cos (\theta))\right\}$,

where $\theta$ and $\varphi$ are the AACGM colatitude and longitude, $P_{l}^{\mathrm{m}}$ are the Legendre polynomial functions, and $c_{l m}$ are complex time-dependent coefficients. Writing $c_{l m}(t)=A_{l m}-i B_{l m}$ in the Eq. (4) and calculating the real part, Eq. 4 can be simplified as follows:

$$
\begin{aligned}
\Phi(\theta, \varphi ; t)= & \sum_{l=0}^{\infty} \sum_{m=0}^{l}\left(A_{l m} \cos (m \phi)+B_{l m} \sin (m \phi)\right) * \\
& * P_{l}^{m}(\cos (\theta)) .
\end{aligned}
$$

Here, the expansion terms have been fully normalised, so that the quantity $\left|c_{l m}\right|^{2}=\left(A_{l m}^{2}+B_{l m}^{2}\right)$ are representative of the variance (mean square value) associated with the component $\{l, m\}$ (Lowes, 1966). Of course, in principle $l$ varies from 0 up to $\infty$ in Eqs. (4) and (6), but in practice the expansion is truncated to a finite order, $L$. The coefficients are evaluated through a least square fit of the function:

$\chi^{2}=\sum_{i=1}^{N} \frac{1}{\sigma_{i}^{2}}\left[\boldsymbol{V}[i] \cdot \hat{\boldsymbol{k}}[i]-W_{i}\right]^{2}$,

where $W_{i}$ and $\sigma_{i}$ are the measured values of the velocity and their standard deviations in the $i$-th grid cell as defined above, while $\boldsymbol{V}[i]$ are the actual vector velocities to be calculated remembering that $\boldsymbol{V}=(\boldsymbol{E} \times \boldsymbol{B}) / B^{2}$ in a quasi collisionless high altitude ionosphere $(>300 \mathrm{~km}$, where most of SuperDARN backscatter comes from), and $\boldsymbol{E}=-\nabla \Phi$, with $\boldsymbol{E}$ and $\boldsymbol{B}$ being the ionospheric electric field and the Earth's magnetic field, respectively. The scalar product in Eq. (6), means that the vector $\boldsymbol{V}[i]$ has to be projected along the direction, $\hat{\boldsymbol{k}}$, where $W_{i}$ has been measured. It has been demonstrated that, even in the presence of quite wide spatial gaps in the data, if the data distribution is uniform through the pattern, the model data do not affect very much the final result, and the overall patterns are really dominated by the measured data (Shepherd and Ruohoniemi, 2000). The velocity vectors $V[i]$ so obtained are always tangent to the equipotential contours. Now, following what reported in Sect. 2 and moving from the expansion of Eq. (4), we introduce a discrete sample space $\Upsilon\{l, m\}$, whose elements are the harmonic functions $\Upsilon_{l, m}=\exp ($ im $\phi)$ $P_{l}^{m}(\cos (\theta))$, and associate to each element of this space a time-dependent number $w_{l, m}(t)$, named relative weight and defined as follows:

$w_{l, m}(t)=\frac{\left|c_{l, m}(t)\right|^{2}}{\sum_{j, k}\left|c_{j, k}(t)\right|^{2}}$,

where $j, l \in[0, L]$, being $L$ the truncation order of the expansion, and $k, m \in[0, L]$. Such a quantity $w_{l, m}(t)$ represents the fraction of the variance (mean square value) of the field $\Phi(\theta, \phi ; t)$ relative to the element $\Upsilon_{l, m}$ at the time $t$, i.e. a measure of the relative relevance of the term $\Upsilon_{l, m}$ at the time $t$. The spectrum of the relative weights $w_{l, m}$ is equivalent to a time-dependent normalised spectrum. Here we limit our analysis only to the investigation of the $l$ spectrum, and instead of the relative weights $w_{l, m}(t)$ we consider: 


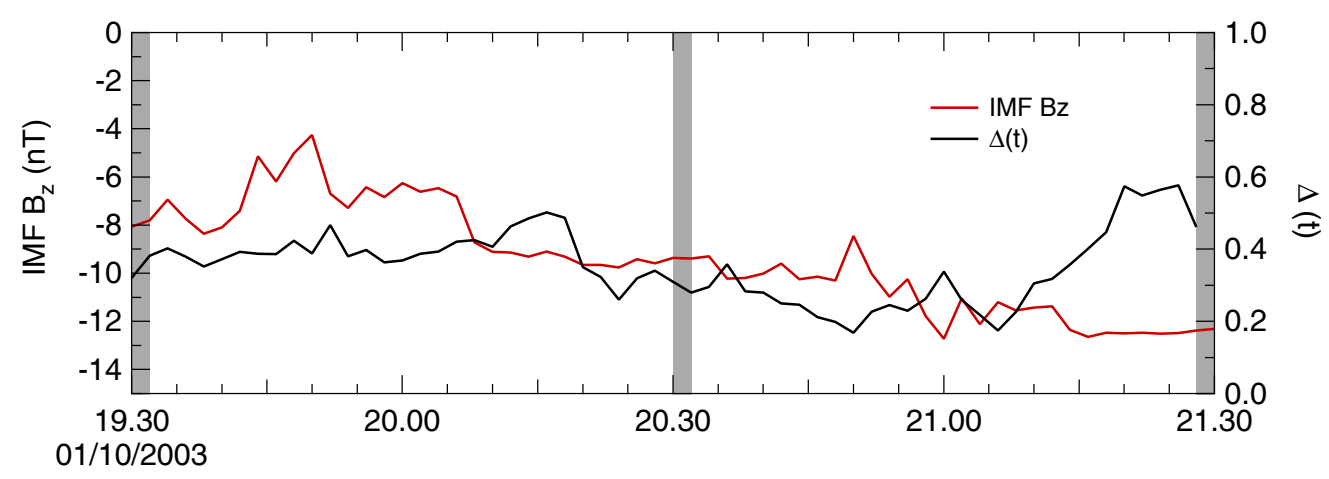

Time (UT)
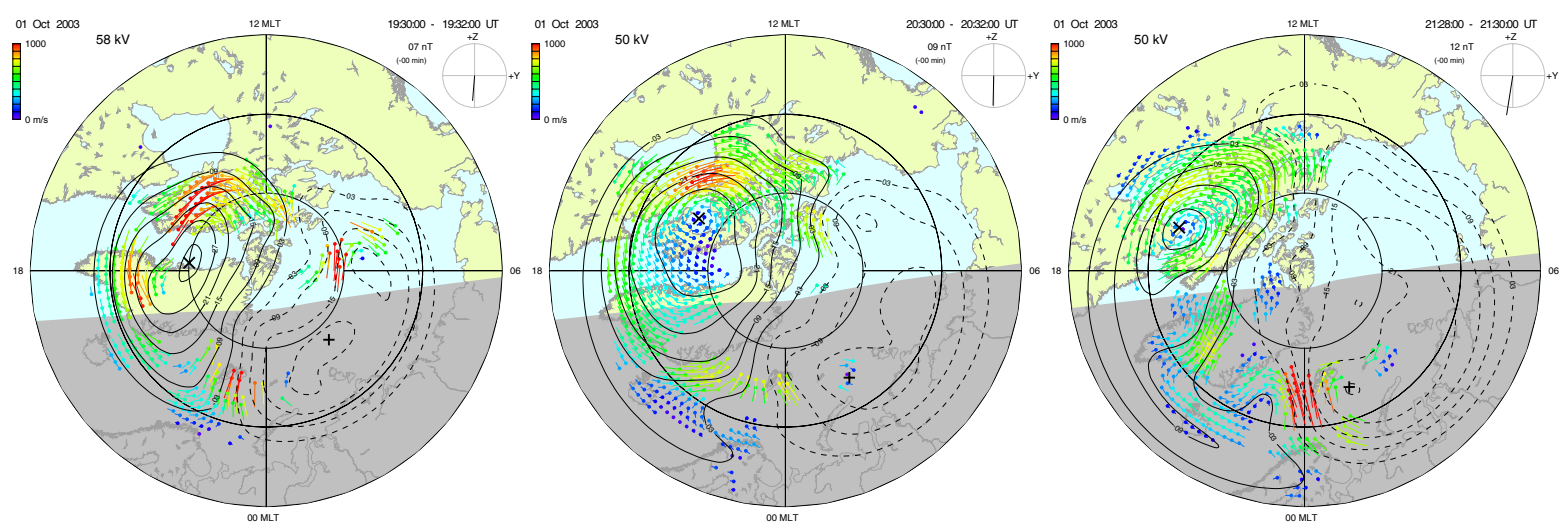

Fig. 1. Upper panel: IMF $B_{z}$ (red, axis on the left) as obtained by OMNI, and normalised disorder degree $\Delta(t)$ (black, axis on the right), for the period 01/10/2003, 19:30-21:30 UT. Lower panel: three samples of SuperDARN convection maps for the same period; the 2-min scan intervals represented in the maps are shaded in the upper panel plot.

$w_{l}(t)=\sum_{m=0}^{l} w_{l, m}(t)=\frac{\sum_{m}\left|c_{l, m}(t)\right|^{2}}{\sum_{j, k}\left|c_{j, k}(t)\right|^{2}}$

where $w_{l}(t)$ is representative of the total relative weight of harmonic functions of degree $l$. From here we can compute the time dependent disorder degree $\Delta(t)$,

$\Delta(t)=-\frac{1}{\log _{2}(L+1)} \sum_{l=0}^{L} w_{l}(t) \log _{2}\left[w_{l}(t)\right]$

and the corresponding time-dependent complexity measure $\Gamma_{11}(t)$,

$\Gamma_{11}(t)=\Delta(t)[1-\Delta(t)]$,

following the definitions given in Sect. 2. According to the given eigenfunction series expansion, the meaning of the quantities $\Delta$ and $\Gamma_{11}$ as measures of disorder and complexity has to be related to the formation of single/multi scale spatial fluctuations. If $\Delta=0$, we are in the presence of a monochromatic spectrum, so that the spatial fluctuations are mainly characterised by a limited set of scales (in the extreme case, by one scale only); conversely, $\Delta=1$ corresponds to a flat spectrum which is associated to multiscale fluctuations. In this framework, complexity shows up in intermediate conditions, i.e. when there is a certain number of fluctuation scales interacting and evolving in time.

\section{Results and discussion}

We have applied the formalism described in the previous Sections to time series of PCP coefficients as obtained from Eqs. (4) and (6) through the Ruohoniemi and Baker (1998) technique. The expansion of the PCP has been limited to the fourth order, as we have checked that the results do not change substantially if we use higher order expansions.

\subsection{Case studies for different IMF orientations}

We first consider two two-hour intervals of SuperDARN data in the Northern Hemisphere, characterised by almost steady conditions of the IMF and good overall coverage.

In Figure 1, upper panel, the IMF $B_{z}$ (red curve) is shown for the 19:30-21:30 UT period on 1 October 2003. IMF data come from the OMNI data base, in GSM coordinates 


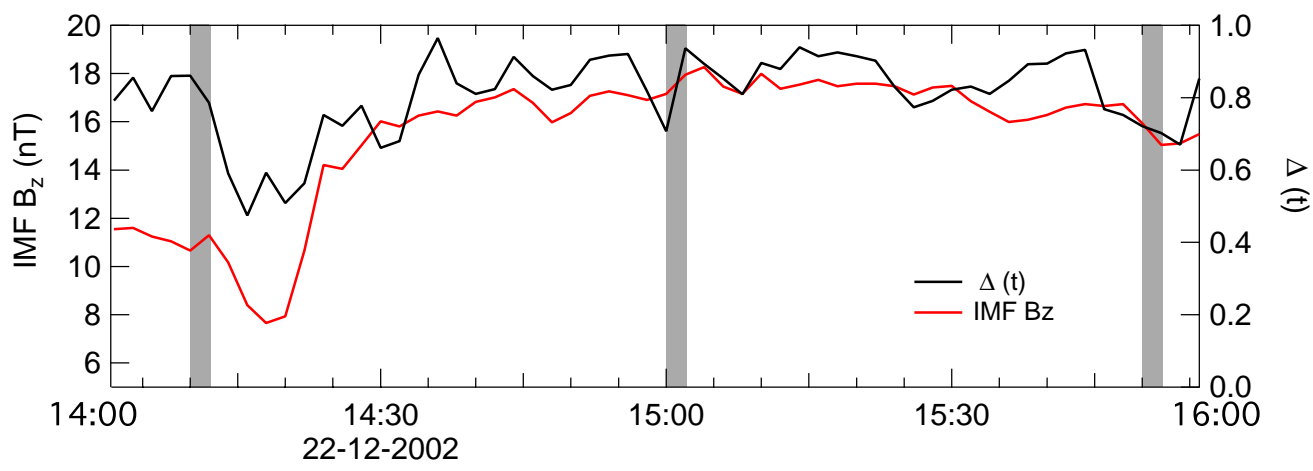

Time (UT)
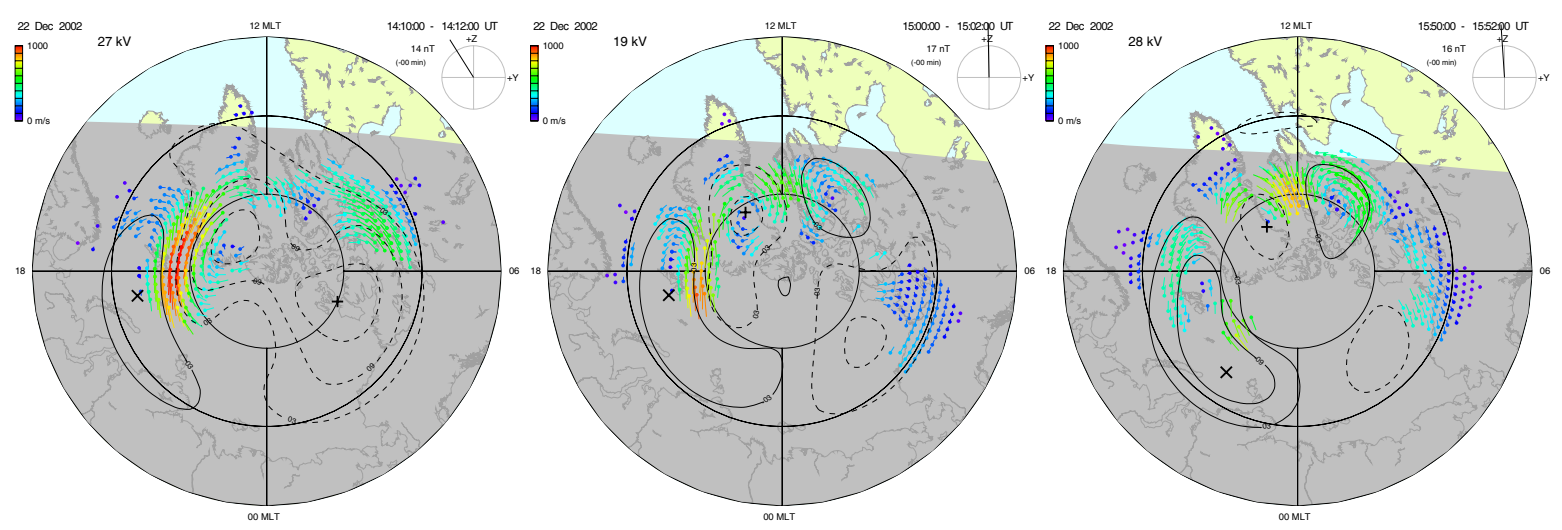

Fig. 2. Upper panel: IMF $B_{z}$ (red, axis on the left), as obtained by OMNI, and normalised disorder degree $\Delta(t)$ (black, axis on the right) for the period 22/12/2002, 14:00-16:00 UT. Lower panel: three samples of SuperDARN convection maps for the same period; the 2-min scan intervals represented in the maps are shaded in the upper panel plot.

in this case, and are resampled in 602 -min bins. The black curve displays the disorder degree $\Delta(t)$ calculated following the procedure described in the previous Section. The lower panel of Fig. 1 displays three 2-min snapshots of convection patterns as obtained from SuperDARN line-of-sight velocity data through the Ruohoniemi and Baker (1998) technique; the scan intervals shown in the snapshots are evidenced in the upper panel plot by gray-shaded areas. This first interval is characterised by a negative IMF $B_{z}$ : from the three patterns taken at the beginning of the period (19:3019:32 UT), at about a half of the period (20:30-20:32 UT) and at the end (21:28-21:30 UT), which are fully representative of the whole event, a steady double cell configuration is evident, typical for such an IMF orientation (Ruohoniemi and Greenwald, 1996, 2005). The average value of $\Delta(t)$ is 0.35 , evidencing that the ionospheric convection is characterised by a low disorder, where the information content contributing to $\Delta(t)$ is concentrated in a few spherical harmonics. After 21:05 UT $\Delta(t)$ starts to rise, reflecting changes in the convection patterns which lead the system towards more complex configurations; in fact, in the last snapshot on the right, in the lower panel of Fig. 1 (21:28-21:30 UT), we can see convection structures appearing around midnight MLT, which perturb the quite regular two-cell symmetry that characterised the convection patterns before 21:00 UT.

Figure 2 shows data, in the same format as in Fig. 1, for the 22 December 2002, 14:00-16:00 UT interval, during which $B_{z}$ was steadily positive. The upper panel displays $B_{z}$ and $\Delta(t)$; the lower panel shows three snapshots of representative convection patterns. Here the convection patterns show a double reverse cell configuration at high latitudes or, in general, strong fluxes of plasma directed sunward in the polar cap; at lower latitudes other convection cells show up, highly variable in size and dynamics. The main difference with respect to Fig. 1 is that $\Delta(t)$ is clearly higher, ranging between 0.6 and 0.9 and attaining the highest values after 14:40 UT, when the IMF $B_{z}$ exceeds $15 \mathrm{nT}$. It is natural to interpret this highly disordered configuration as an evidence of the contribution of a greater number of harmonics to the formation of the convection pattern. Moreover, we notice a qualitative correlation between $\Delta(t)$ and $B_{z}$. Figure 3, upper panel, shows normalised histograms of $\Delta$ for all data points pertaining to Figs. 1 and 2, in red and blue for the scans of Figs. 1 and 2, respectively. The two $\Delta$ sets for negative and positive $B_{z}$ are clearly separated, with only a small overlap around $\Delta=0.5$. The negative $B_{z}$ population peaks between 0.3 and 0.4 and 


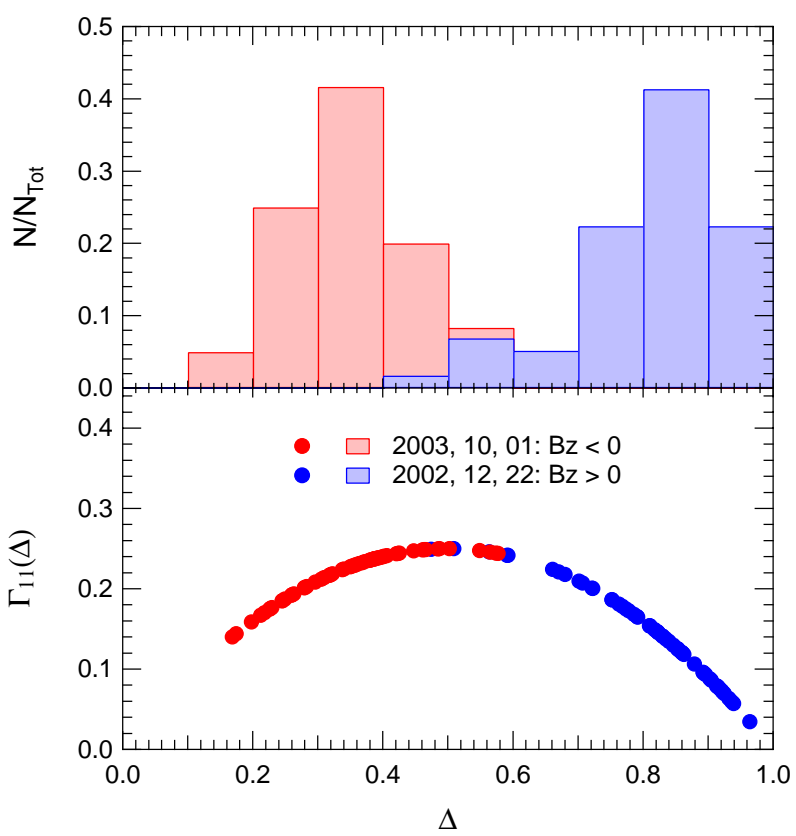

Fig. 3. Top panel: histograms of the normalised disorder degree $\Delta$, in 0.1 bins, for the 1 October 2003, 19:30-21:30 UT (IMF $B_{z}<0$, red cityscape) and 22 December 2002, 14:00-16:00 UT (IMF $B_{z}>0$, blue cityscape) intervals; the values of each histogram have been normalised to the total number of scans (which is 60 for both). Bottom panel: second order complexity measure $\Gamma_{11}$ as a function of the normalised disorder degree $\Delta$ for the same time intervals (IMF $B_{z}<0$ : red dots; IMF $B_{z}>0$ : blue dots).

$93 \%$ of its points fall below $\Delta=0.5$, which means that in these cases the system tends to a more ordered configuration; on the other hand, the positive $B_{z}$ population peaks between 0.8 and 0.9 and $98 \%$ of its points exceed 0.5 , which corresponds to a high desorder degree. In the lower panel of Fig. 3 we show $\Gamma_{11}$ plotted against $\Delta$ : red dots represent pairs of $\Delta$ and $\Gamma_{11}$ values for the $B_{z}<0$ interval, while blue dots refer to the scans of the $B_{z}>0$ interval. As expected from its definition, the maximum complexity, $\Gamma_{11}=0.25$ is attained around $\Delta \simeq 0.5$, i.e. corresponding to intermediate values of the normalised disorder parameter $\Delta$. This presentation emphasizes the fact that complexity is low both for high and for low values of $\Delta$, which correspond to more disordered and more ordered convection configurations, respectively.

Around the complexity maximum, we find convection patterns which correspond to both positive and negative IMF $B_{z}$.

Figure 4 displays the time series of IMF $B_{z}$ (red curve) and the time series of $\Delta$ (black curve) for a period, 19 December 2002, 06:00-10:00 UT, characterised by a variable IMF $B_{z}$. In fact, $B_{z}$ was positive until about 07:32 UT, then became negative and switched again to positive values at about 08:52 UT. The values of $B_{z}$ spanned from -15 up to $16 \mathrm{nT}$. Two vertical dashed lines mark the reversals of the sign of $B_{z}$ in Fig. 4. One can see a correlation between the two curves:

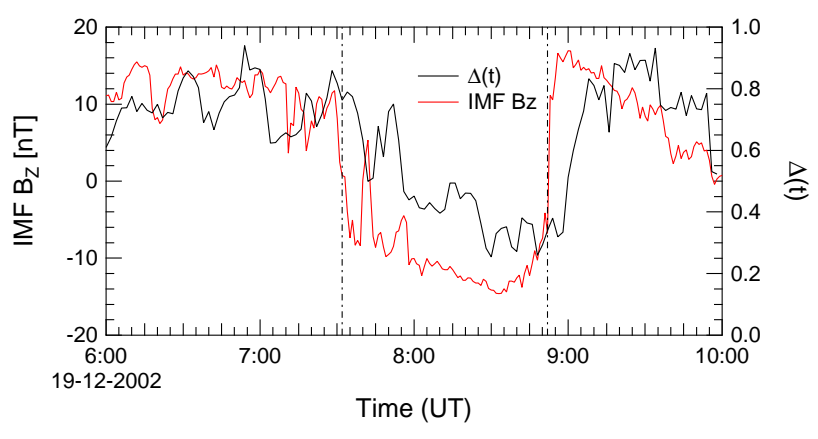

Fig. 4. Time series of IMF $B_{z}$ (red, axis on the left) in GSM coordinates, and normalised disorder degree $\Delta(t)$ (black, axis on the right) for the 19 December 2002, 06:00-10:00 UT interval. The vertical black dashed lines indicates the times when $B_{z}$ changes of sign, from positive to negative and vice versa.

$\Delta$ closely follows the IMF $B_{z}$ behaviour, reaching high or intermediate values when $B_{z}$ is positive (maximum disorder), and taking lower values when $B_{z}$ is negative (maximum order). We also notice that the $\Delta$ curve exhibits a lag relative to the $B_{z}$ curve, which is particularly evident immediately after the $B_{z}$ rotations. By cross-correlating the two time series we have found that this time lag amounts to $10 \mathrm{~min}$. Therefore, before building histograms as in the upper panel of Fig. 3, we time-lagged the $B_{z}$ data by 10 min (not shown).

Figure 5, upper panel, shows such histograms for the 19 December 2002, 06:00-10:00 UT event, in the same format used for Fig. 3, i.e. in red and blue for the positive and negative $B_{z}$ scans, respectively. In this case, the negative $B_{z}$ population peaks between 0.4 and 0.5 and $70 \%$ of its points fall below $\Delta=0.5$, corresponding to the system collapsing towards order, while the positive $B_{z}$ population peaks between 0.7 and 0.8 and $100 \%$ of its points exceed 0.5 , which corresponds to a high desorder degree. We can conclude that, in this case too, the $\Delta$ histograms for positive and negative $B_{z}$ are clearly separated, although the overlap of the two populations is somewhat greater than in the case of Fig. 3 and is slightly shifted toward $\Delta>0.5$.

Figure 5, lower panel, shows $\Gamma_{11}$ as a function of $\Delta$ for the same interval: again red dots represent pairs of $\Delta$ and $\Gamma_{11}$ values for the scans characterised by negative IMF $B_{z}$, while blue dots are for pairs of $\Delta$ and $\Gamma_{11}$ for positive $B_{z}$ scans. Like for the two intervals shown in Fig. 3, lower panel, also in this case we observe a mixing of ordered and disordered configurations across the maximum of $\Gamma_{11}$. Indeed, several points representing negative $B_{z}$ configurations are rather found on the right side of the curve, where the disorder degree is high. In this regard, we may add that, by careful visual inspection, we have found that such scans are mostly the ones closest to the changes of sign of $B_{z}$ and often correspond to $B_{z}$ values close to zero, both positive and negative. Having said all that, we can consider the possibility that other 


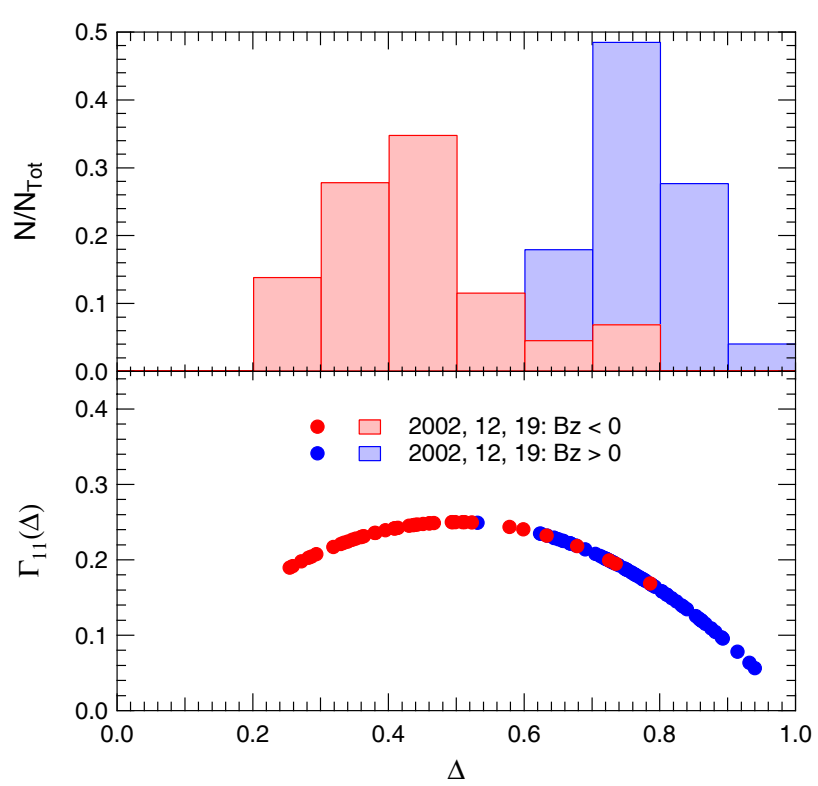

Fig. 5. Upper Panel: normalised histograms of the normalised disorder degree $\Delta$, in 0.1 bins, for the 19 December 2002, 06:0010:00 UT interval; red and blue cityscapes correspond to negative and positive IMF $B_{z}$ scans, respectively. Values have been normalised to the total number of scans for each $B_{z}$ polarity (69 and 46 for positive and negative values, respectively). Lower panel: second order complexity measure $\Gamma_{11}$ as a function of the normalised disorder degree $\Delta$ for same time interval.

parameters than $B_{z}$ may be at play in determining the distribution of scan occurrence as a function of $\Delta$. In this regard, the first possible candidate is obviously IMF $B_{y}$, given its non-negligible role in the formation of ionospheric convection cells. Actually, $B_{y}$ does exhibit some variations in the time interval corresponding to Fig. 4. However, a 115 points statistics (about $4 \mathrm{~h}$ of 2-min radar scans) is too limited for a reliable characterisation of the relative roles of $B_{z}$ and $B_{y}$. Hence, we perform such a study in the next Section through the analysis of a much longer time period.

\subsection{Study of an extended time interval}

In this Section we will make use of a larger statistical sample of data in order to investigate the combined influence of IMF $B_{z}$ and $B_{y}$ on both complexity, $\Gamma_{11}$, and disorder degree, $\Delta$. For that purpose, we selected a period of twenty days of SuperDARN data in the Northern Hemisphere, during February 2002, on the grounds that this particular month is characterised by an abundant and almost uniform data coverage and the IMF and the solar wind show a wide variety of conditions. The time series of the three periods described in the previous Section have been added to the sample as well.

For each of the 13604 SuperDARN 2-min scans pertaining to the selected period, we calculated the 4th-order PCP
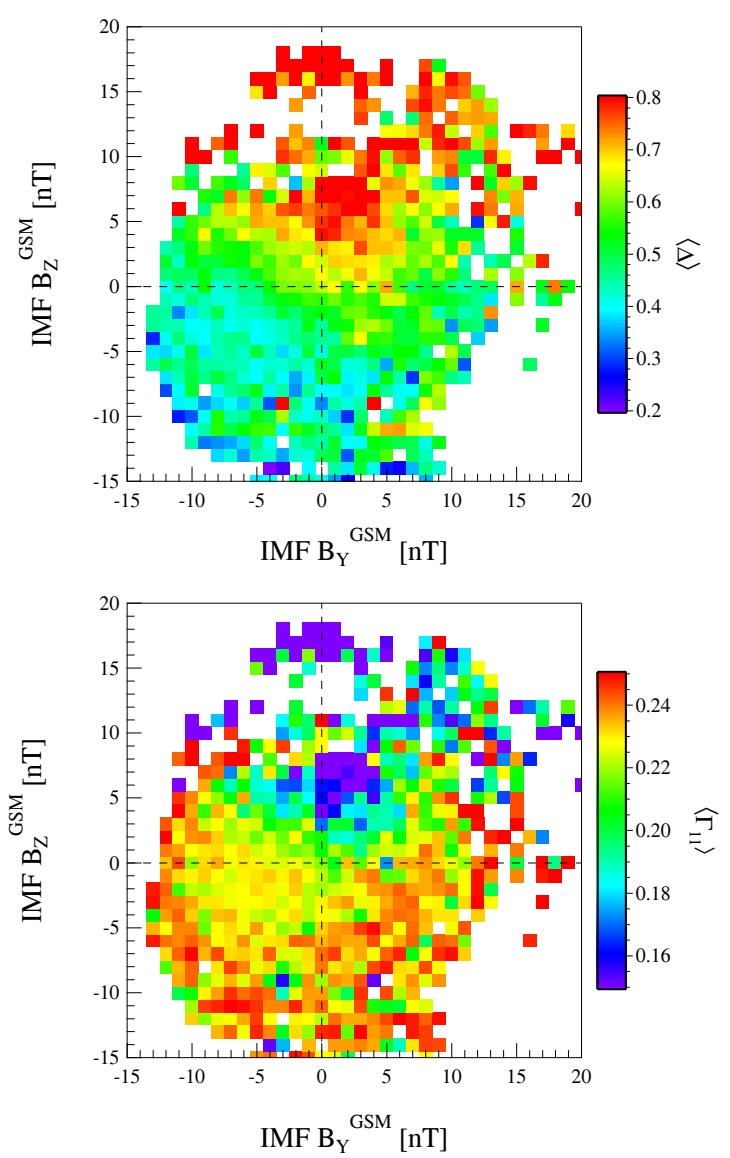

Fig. 6. February 2002: $\langle\Delta\rangle$ (upper panel) and $\left\langle\Gamma_{11}\right\rangle$ (lower panel) as functions of $B_{y}$ and $B_{z}$. The colour scale for each function is displayed on the right of each panel.

coefficients and then calculated the $\Delta$ and $\Gamma_{11}$ parameters. As a second step of the analysis, we calculated averages of $\Delta$ and $\Gamma_{11}\left(\langle\Delta\rangle,\left\langle\Gamma_{11}\right\rangle\right)$ in $\left[B_{z}, B_{y}\right]$ two dimensional bins $1 \times 1 \mathrm{nT}$ wide, from -15 up to $20 \mathrm{nT}$ for both $B_{z}$ and $B_{y}$. In order to avoid as much as possible the effects of time lags like the one described in the case of Fig. 4, the daily time series of the IMF $B_{z}$ have been cross-correlated with the corresponding $\Delta$ time series and daily time lags have been determined and applied to the $B_{z}$ data. The average of such lag times is $16 \mathrm{~min}$ ( $\pm 3 \mathrm{~min})$. In order to exclude data with a too low statistical relevance, we dropped all averages pertaining to bins containing less than 10 scans. Figure 6 shows colour coded plots of such averages of $\langle\Delta\rangle$ (upper panel) and $\left\langle\Gamma_{11}\right\rangle$ (lower panel) as a function of $B_{z}$ and $B_{y}$. The results confirm and extend those for the case studies discussed above.

When $B_{z}$ is negative, the complexity measure is generally high (above 0.22 almost everywhere), assuming the highest values when $B_{y}$ is dominant over $B_{z}$. One could expect lower values of $\langle\Delta\rangle$ and $\left\langle\Gamma_{11}\right\rangle$ when $B_{z}$ is negative and dominant over $B_{y}$, as we observed for example in the time period shown in Fig. 1; in this respect, we must say that the extended 
time period we have chosen does not contain very long and steady intervals of strong negative $B_{z}$, so that we can speculate that the system never really finds the favourable conditions for an "ideal" Dungey cycle activation. In such a case, a stable two-cell configuration should confine the information content in few harmonics, and the nearly maximum order for the system would be realised. In a more realistic picture, frequent $B_{z}$ fluctuations continuously force the system in non-long standing stationary states, so increasing disorder and complexity.

The complexity decreases when $B_{z}$ turns from negative to positive values: the ionospheric convection during positive $B_{z}$ periods tends to configurations which show a strong topological disorder. Moreover, a broad region is evident for $0<B_{z}<10 \mathrm{nT}$ and $\left|B_{y}\right| / B_{z}<1$, where $0.6<\langle\Delta\rangle<0.9$ and complexity is low. We note also that in the same $B_{z}$ domain the increase of $\left|B_{y}\right|$ tends to reduce the disorder and increase the complexity. Furthermore, a certain asymmetry in response to increasing IMF $B_{y}$ is observed: for positive IMF $B_{z},\langle\Delta\rangle$ and $\left\langle\Gamma_{11}\right\rangle$ seem to increase more when $B_{y}>0$.

One can conclude that, on a statistical basis, although the IMF $B_{z}<0$ dominates the transition towards a more ordered $(\langle\Delta\rangle<0.5)$ and complex configuration of the ionospheric convection, a similar effect is also due to IMF $B_{y}$ when $\left|B_{y}\right| / B_{z} \gg 1$.

\section{Conclusions}

In this work we studied the reconfiguration of ionospheric convection from the point of view of information theory and complex system physics, so far not applied to such an issue. Starting from the Polar Cap Potential coefficients, as obtained from SuperDARN convection velocity data, we quantitatively computed the pseudo Shannon entropy, the disorder degree and the degree of complexity associated with the PCP structure on a global scale for three "paradigm" intervals first, and for an extended time period of about twenty days of data.

The obtained results clearly evidenced how the degree of complexity is a function of the IMF configuration. Indeed, a clear signature of a reduction of disorder and an increase of complexity is found when IMF turns from northward to southward. This behaviour can be interpreted in terms of a dynamical phase transition of the ionospheric convection pattern topology. Furthermore, when $\left|B_{y}\right| / B_{z} \gg 1$, a similar effect was found as a function of the IMF $B_{y}$ intensity, so that both $B_{z}$ and $B_{y}$ may be regarded as acting as control parameters.

The observed decrease of disorder for southward IMF $B_{z}$ has to be related to the emergence of a large scale coherence in the PCP structure manifesting in a more simple nearly two-cell structure. Conversely, the higher degree of disorder for northward IMF $B_{z}$ conditions reflects the inherent small scale multi-cell structure of ionospheric convection, which has to be associated with a reduced coherence in the large scale convection motions. It is in this framework that the transition from the small scale multi-cell structure of ionospheric convection for northward IMF condition to the nearly two-cell structure observed for southward IMF $B_{z}$ is read as a dynamical order-disorder topological phase transition, monitored by the changes in $\Delta$ and $\Gamma_{11}$.

In the recent literature (e.g. Sharma and Kaw, 2005; Consolini et al., 2008) it has been evidenced how the overall magnetospheric dynamics is well in agreement with that of a system near a nonequilibrium stationary state displaying dynamical complexity. In such a scenario, the topological phase transition, occurring during the increase of the global magnetospheric convection due to a southward turning of the IMF condition, is analogous to what occurs, for instance, in the case of Rayleigh-Bénard convection, when a long range coherence emerges out-of-equilibrium at high values of the overall temperature gradient and it is observed a reduction in the symmetry degree of the system. Paraphrasing the last concepts, we could say that the emergence of a long range coherence in the convection pattern during the southward turning of the IMF $B_{z}$ component is a manifestation of a first-order like phase transition accompanied by a symmetrybreaking phenomenon.

The qualitative correlation between the $\Delta(t)$ and IMF $B_{z}$ time series, shown in Figs. 1, 2 and 4, and the apparent systematic delay between the two curves deserve further investigation in future studies, in order to explore the possibility of using $\Delta$ as a "quicklook" parameter of the overall ionospheric convection.

Acknowledgements. This work is supported by the Italian Space Agency (ASI) through the Exploration of Space System 2 program. The authors kindly aknowledge N. Papitashvili and J. King at the National Space Science Data Center of the Goddard Space Flight Center for the use permission of the 1-min IMF OMNI data and the NASA CDAWeb team for making these data available.

Edited by: P.-L. Sulem

Reviewed by: M. Leubner, Y. Elskens, and another anonymous referee

\section{References}

Abel, G. A., Freeman, M. P., and Chisham, G.: IMF clock angle control of multifractality in ionospheric velocity fluctuations, Geophys. Res. Lett., 36, L19102, doi:10.1029/2009GL040336, 2009.

Axford, W. I. and Hines, C. O.: A unifying theory of high-latitude geophysical phenomena and geomagnetic storms, Can. J. Phys., 39, 1433-1464, 1961.

Baker, K. B. and Wing, S.: A new magnetic coordinate system for conjugate studies at high latitudes, J. Geophys. Res., 94, 91399143, 1989.

Burke, W. J., Kelley, M. C., Sagalyn, R. C., Smiddy, M., and Lai, S. T.: Polar cap electric field structures with a northward interplanetary magnetic field, Geophys. Res. Lett., 6, 21-24, 1979. 
Chang, T.: Low dimensional behaviour and symmetry breaking of stochastic systems near criticality - Can these effects be observed in space and in the laboratory?, IEEE T. Plasma Sci., 20, 691694, 1992

Chang, T., Tam, S. W. Y., and Wu, C.-C.: Complexity in space plasmas - a brief review, Space Sci. Rev., 122, 281-291, 2006.

Chen, J., Sharma, A. S., Edwards, J. W., Shao, X., and Kamide, Y: Spatiotemporal dynamics of the magnetosphere during geospace storms: Mutual information analysis, J. Geophys. Res., 113, A05217, doi:10.1029/2007JA012310, 2008.

Chisham, G., Lester, M., Milan, S. E., Freeman, M. P., Bristow, W. A., Grocott, A., McWilliams, K. A., Ruohoniemi, J. M., Yeoman, T. K., Dyson, P. L., Greenwald, R. A., Kikuchi, T., Pinnock, M., Rash, J. P. S., Sato, N., Sofko, G. J., Villain, J.-P., and Walker, A. D. M.: A decade of the Super Dual Auroral Radar Network (SuperDARN): scientific achievements, new techniques and future directions, Surv. Geophys., 28, 33-109, 2007.

Chisham, G., Freeman, M. P., Abel, G. A., Lam, M. M., Pinnock, M., Coleman, I. J., Milan, S. E., Lester, M., Bristow, W. A., Greenwald, R. A., Sofko, G. J., and Villain, J.-P.: Remote sensing of the spatial and temporal structure of magnetopause and magnetotail reconnection from the ionosphere, Rev. Geophys., 46, RG1004, doi:10.1029/2007RG000223, 2008.

Consolini, G., Marcucci, M. F., and Candidi, M.: Multifractal structure of auroral electrojet index data, Phys. Rev. Lett., 76, 40824085, 1996.

Consolini, G., De Michelis, P., and Tozzi, R.: On the Earth's magnetospheric dynamics: Nonequilibrium evolution and the fluctuation theorem, J. Geophys. Res., 113, A08222, doi:10.1029/2008JA013074, 2008.

Consolini, G., Tozzi, R., and De Michelis, P.: Complexity in the sunspot cycle, Astron. Astrophys., 506, 1381-1391, 2009.

Cowley, S. W. H., and Lockwood, M.: Excitation and decay of solar wind-driven flows in the magnetosphere-ionosphere system, Ann. Geophys., 10, 103-115, 1992, http://www.ann-geophys.net/10/103/1992/.

De Michelis, P., Consolini, G., Materassi, M., and Tozzi, R.: An information theory approach to storm-substorm relationship, J. Geophys. Res., 116, A08225, doi:10.1029/2011JA016535, 2011.

De Santis, A., Tozzi, R., and Gaya-Piqué, L. R.: Information content and K-entropy of the present geomagnetic field, Earth Planet. Sci. Lett., 218, 269-275, 2004.

Dungey, J. W.: Interplanetary magnetic field and the auroral zones, Phys. Rev. Lett., 6, 47-50, 1961.

Ebeling, W. and Klimontovich, Y. L. (Eds.): Self-organization and turbulence in liquids, Teubner-Verlag, Leipzig, Germany, 1984.

Feller, W.: An introduction to probability theory and its applications, 3rd Edition, Vol. I, J. Wiley \& Sons Inc., USA, 1970.

Gosling, J. T., Thomsen, M. F., Bame, S. J., Elphic, R. C., and Russell, C. T.: Plasma flow reversals at the dayside magnetopause and the origin of asymmetric polar cap convection, J. Geophys. Res., 95, 8073-8084, 1990.

Greenwald, R. A., Baker, K. B., Dudeney, J. R., Pinnock, M., Jones, T. B., Thomas, E. C., Villain, J.-P., Cerisier, J.-C., Senior, C., Hanuise, C., Hunsucker, R. D., Sofko, G., Koelher, J., Nielsen, E., Pellinen, R., Walker, A. D. M., Sato, N., and Yamagishi, Y.: DARN/SUPERDARN: A Global View of the Dynamics of HighLatitude Convection, Space Sci. Rev., 71, 761-796, 1995.

Haken, H.: Synergetics, Introduction and Advanced Topics,
Springer-Verlag, Berlin, 2004.

Huang, C.-S., Andre, D. A., Sofko, G. J., and Koustov, A. V.: Super Dual Auroral Radar Network observations of ionospheric multicell convection during northward interplanetary magnetic field, J. Geophys. Res., 105, 7419-7428, 2000.

Klimas, A. J., Vassiliadis, D., Baker, D. N. and Roberts, D. A.: The organized nonlinear dynamics of the magnetosphere, J. Geophys. Res., 101(A6), 13089-13113, doi:10.1029/96JA00563, 1996.

Klimontovich, Yu. L.: Turbulent Motion and the Structure of Chaos, A new Approach to the Statistical Theory of Open Systems, Kluwer Academic Publishers, Dordrecht, The Netherlands, 1991.

Klimontovich, Yu. L.: Criteria of Self-organization, Chaos Solut. Fractals, 5, 1985-2002, 1995.

Klimontovich, Yu. L.: Relative ordering criteria in open systems, Physics-Uspekhi, 39, 1169-1179, 1996.

Landsberg, P. T.: Thermodynamics and statistical mechanics, Oxford University Press, London, 1978.

Landsberg, P. T.: Can entropy and order increase togheter?, Phys. Lett. A, 102, 171-173, 1984.

Landsberg, P. T. and Shiner, J. S.: Disorder and complexity in an ideal non-equilibrium Fermi gas, Phys. Lett. A, 245, 228-232, 1998.

Lowes, F. J.: Mean-square values on sphere of spherical harmonic vector fields, J. Geophys. Res., 71, p. 2179, 1966.

Nicolis, G. and Nicolis, C: Foundations of Complex Systems, Nonlinear Dynamics, Statistical Physics, Information and Prediction, World Scientific Publishing, Singapore, 2007.

Parkinson, M. L.: Dynamical critical scaling of electric field fluctuations in the greater cusp and magnetotail implied by HF radar observations of F-region Doppler velocity, Ann. Geophys., 24, 689-705, 2006, http://www.ann-geophys.net/24/689/2006/.

Parkinson, M. L.: Complexity in the scaling of velocity fluctuations in the high-latitude F-region ionosphere, Ann. Geophys., 26, 2657-2672, doi:10.5194/angeo-26-2657-2008, 2008.

Quian Quiroga, R., Arnhold, J., Lehnertz, K., and Grassberger, P.: Kulback-Leinler and renormalized entropis: Applications to electroencephalograms of epilepsy patients, Phys. Rev. E, 62, 8380-8386, 2000.

Reiff, P. H. and Burch, J. L.: IMF By-dependent plasma flow and Birkeland currents in the dayside magnetosphere 2, A global model for northward and southward IMF, J. Geophys. Res., 90, 1595-1609, 1985.

Ruohoniemi, J. M. and Baker, K. B.: Large-scale imaging of highlatitude convection with Super Dual Auroral Radar Network HF radars observations, J. Geophys. Res., 103, 20797-20811, 1998.

Ruohoniemi, J. M. and Greenwald, R. A.: Statistical patterns of high-latitude convection obtained from Goose Bay HF radar observations, J. Geophys. Res., 101, 21743-21763, 1996.

Ruohoniemi, J. M. and Greenwald, R. A.: Dependencies of high-latitude plasma convection: Consideration of interplanetary magnetic field, seasonal, and universal time factors in statistical patterns, J. Geophys. Res., 110, A09204, doi:10.1029/2004JA010815, 2005.

Sello, S.: Wavelet entropy as a measure of solar cycle complexity, Astron. Astrophys., 363, 311-315, 2000.

Sello, S.: Wavelet entropy and the multi-peaked structure of solar cycle maximum, New Astron., 8, 105-117, 2003. 
Shannon, C. E.: A mathematical theory of communication, Bell Syst. Tech. J., 27, 379-423, 1948.

Sharma, A. S. and Kaw, P. K. (Eds.): Nonequilibrium phenomena in plasmas, Springer, Dordrecht, The Netherlands, 2005.

Shepherd, S. G. and Ruohoniemi, J. M.: Electrostatic potential patterns in the high latitude ionosphere constrained by SuperDARN measurements, J. Geophys. Res., 105, 23005-23014, 2000.

Shiner, J. S., Davison, M., and Landsberg, P. T.: Simple measure of complexity, Phys. Rev. E, 59, 1459-1464, 1999.

Sitnov, M. I., Sharma, A., Papadopoulos, K., Vassiliadis, D., Valdivia, J., Klimas, A., and Baker, D.: Phase transition-like behavior of the magnetosphere during substorms, J. Geophys. Res., 105, 12955-12974, 2000.
Sitnov, M. I., Sharma, A., Papadopoulos, K., and Vassiliadis, D.: Modeling substorm dynamics of the magnetosphere: From self-organization and self-organized criticality to nonequilibrium phase transitions, Phys. Rev. E, 65, 016116, doi:10.1103/PhysRevE.65.016116, 2001.

Wicks, R. T., Chapman, S. C., and Dendy, R. O.: Mutual information as a tool for identifying phase transition in dynamical complex systems with limited data, Phys. Rev. E, 75, 051125, doi:10.1103/PhysRevE.75.051125, 2007. 